\title{
Desarrollo e Implementación de Técnicas y Dispositivos para la Aplicación de Electroporación como Tratamiento Tumoral
}

\author{
Borja López-Alonso, Héctor Sarnago, Óscar Lucía y José M. Burdío \\ Grupo de Electronica de Potencia y Microelectronica (GEPM) \\ Instituto de Investigación en Ingeniería de Aragón (I3A) \\ Universidad de Zaragoza, Mariano Esquillor s/n, 50018, Zaragoza, Spain. \\ Tel.+34-976762707, e-mail: blopez@unizar.es
}

\section{Resumen}

La electroporación es un fenómeno que aumenta la permeabilidad de las membranas celulares mediante pulsos de campo eléctrico. Entre sus aplicaciones, la ablación tumoral presenta importantes ventajas derivadas de la ausencia de efectos térmicos. Este artículo detalla los dispositivos y técnicas desarrollados que permiten optimizar y controlar dichos tratamientos.

\section{Introducción}

La electroporación dentro del campo de la medicina tiene grandes aplicaciones en oncología. En función de la intensidad del campo eléctrico aplicado, la permeabilización de la membrana será de carácter reversible o irreversible. Si se aplica un campo de baja intensidad se produce electroporación reversible (RE). Esta es utilizada para aumentar la absorción focalizada de medicamentos quimioterápicos, dando lugar a la electroquimioterapia. Si el campo aplicado es de alta intensidad, se producen lesiones irreversibles en las membranas que conducen a la muerte de las células. Este fenomeno se conoce como electroporación irreversible (IRE) y se utiliza como método de ablacion tumoral no térmico. A diferencia de los tratamientos tumorales actuales, la electroporación permite tratar tejidos con alta perfusión sanguínea, no produce lesiones de carácter térmico, y permite preservar las estructuras de los vasos sanguíneos aumentando el volumen de tejido preservado, siendo una alternativa muy interesante para aquellos casos en los que no es posible aplicar tratamientos quirúrgicos o térmicos convencionales.

\section{Motivación y Objetivos}

En la actualidad los dispositivos comerciales que existen para aplicar estos tratamientos tienen prestaciones reducidas en cuanto a potencia de salida y flexibilidad en los protocolos de aplicación [1]. Estos dispositivos tampoco permiten realizar control en tiempo real del tratamiento. Por todo esto es necesario el desarrollo de nuevos dispositivos, técnicas y protocolos que permitan realizar tratamientos más eficaces y de manera más segura, logrando así una electroporación focalizada y homogénea. Debido al carácter multidisciplinar de la investigación, se ha llevado a cabo una colaboración entre distintos grupos internacionales [2-4] que pertenecen a las áreas de: Ingeniería Electrónica, Histopatología, Cirugía y Biología. Dicha colaboración permite el desarrollo de nuevos equipos y su evaluación en condiciones clínicas reales. También ha permitido el planteamiento y estudio en profundidad de mejoras en los protocolos de aplicación de los tratamientos [5].

\section{Resultados}

Se han obtenido resultados a diferentes niveles, tal como se muestra en la Fig. 1. Se ha llevado a cabo el desarrollo de tres generaciones de dispositivos de electroporación. La primera generación permitió alcanzar los $10 \mathrm{KV}$ y 200 A de salida, logrando así hacer posible el tratamiento de mayores volúmenes de tejido [6]. Estos dispositivos se han evolucionado hasta la generacion representada en la Fig. 2. Ésta permite llevar a cabo el tratamiento ejerciendo control mediante la monitorización continua de la impedancia en pequeña señal. También se ha logrado la aplicación de un campo eléctrico de orientación variable mediante el desarrollo de electrodos matriciales y un generador 18 salidas conmutadas.

Fruto de la colaboracion se profundizado en el estudio de los efectos de la electroporación a nivel fisiológico y estructural [7-10]. También se ha investigado la focalización del tratamiento alterando la conductividad de los tejidos in vivo. Se ha colaborado en el estudio de la diferenciación celular controlada mediante las oscilaciones de calcio en células madre.

\section{Conclusiones}

Durante esta investigación, y tal como se muestra en la Fig. 1, se han desarrollado hasta 3 generaciones de dispositivos de electroporación, siendo estos puestos a prueba en entornos clínicos. Gracias a ellos se han evaluado en profundidad los efectos a largo plazo del

Revista “Jornada de Jóvenes Investigadores del I3A”, vol. 8 (Actas de la IX Jornada de Jóvenes Investigadores del I3A -11 de diciembre de 2020). ISSN 2341-4790. 
tratamiento, y se ha diseñado un prototipo que incorpora todos los avances obtenidos durante la investigación.

\section{REFERENCIAS}

[1] C. Bernal et al., "A Review of Pulse Generation Topologies for Clinical Electroporation," in Iecon 2015 - 41st Annual Conference of the Ieee Industrial Electronics Society(IEEE Industrial Electronics Society, New York: Ieee, 2015, pp. 625-630.

[2] T. Garcia-Sanchez et al., "Successful Tumor Electrochemotherapy Using Sine Waves," (in English), Ieee Transactions on Biomedical Engineering, Article vol. 67, no. 4, pp. 1040-1049, Apr 2020.

[3] O. Lucia, H. Sarnago, T. Garcia-Sanchez, L. M. Mir, and J. M. Burdio, "Industrial Electronics for Biomedicine A New Cancer Treatment Using Electroporation," (in English), Ieee Industrial Electronics Magazine, Article vol. 13, no. 4, pp. 618, Dec 2019.

[4] H. Sarnago, J. M. Burdio, T. Garcia-Sanchez, L. M. Mir, I. Alvarez-Gariburo, and O. Lucia, "GaN-Based Versatile Waveform Generator for Biomedical Applications of Electroporation," (in English), Ieee Access, Article vol. 8, pp. 97196-97203, 2020.
[5] B. López-Alonso, H. Sarnago, J. M. Burdío, and O. Lucía, "Electro-thermal modeling of irreversible electroporation and validation method of electric field distribution," International Journal of Applied Electromagnetics and Mechanics, vol. 63, pp. 41-50, 2020.

[6] H. Sarnago, L. Ó, A. Naval, J. M. Burdío, Q. Castellví, and A. Ivorra, "A Versatile Multilevel Converter Platform for Cancer Treatment Using Irreversible Electroporation," IEEE Journal of Emerging and Selected Topics in Power Electronics, vol. 4, no. 1, pp. 236-242, 2016.

[7] B. López-Alonso et al., "Histopathological and Ultrastructural Changes after Electroporation in Pig Liver Using Parallel-Plate Electrodes and High-Performance Generator," Scientific Reports, vol. 9, no. 1, pp. 1-12, 2019/02/25 2019.

[8] P. Iruzubieta et al., "Ultrastructural study of pig liver regeneration following ablation with irreversible electroporation," "Histology and Histopathology, vol. 34, p. 137, 2019.

[9] M. C. Junquera et al., "Electron microscope evaluation of irreversible electroporation on the liver in a porcine model," Histology and histopathology, vol. 32, p. 125, 2017.

[10] E. Monleón et al., "Remodelling of liver tissue after irreversible electroporation in porcine," Histology and Histopathology, vol. 34, p. 138, 2019.

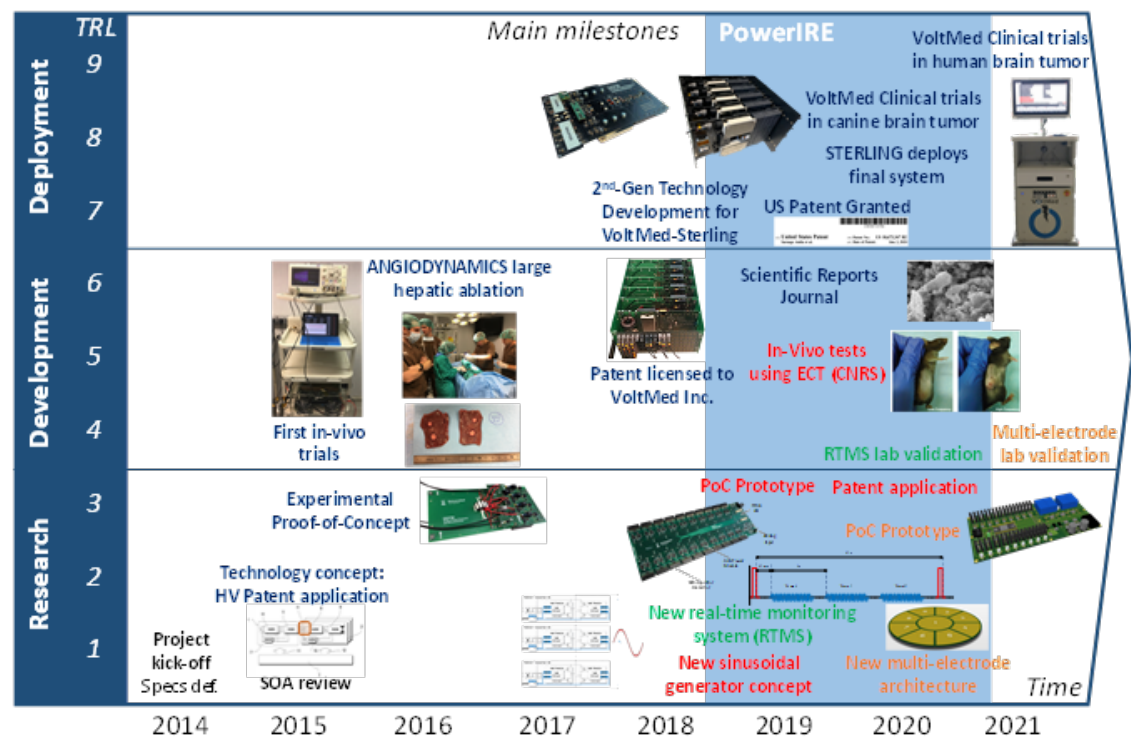

Fig. 1 Figura resumen de los avances realizados.

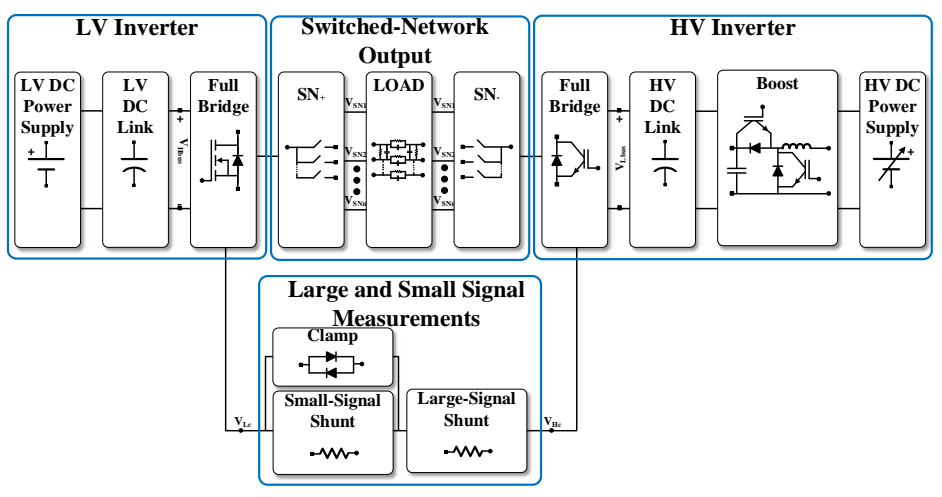

(a)

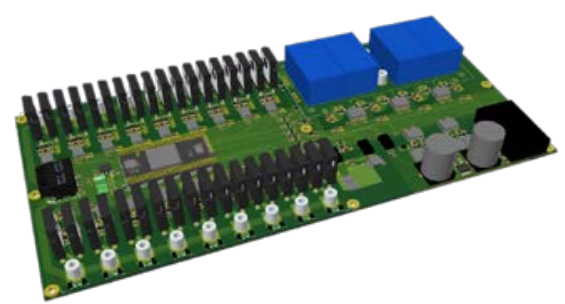

(b)

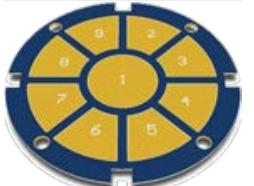

(c)

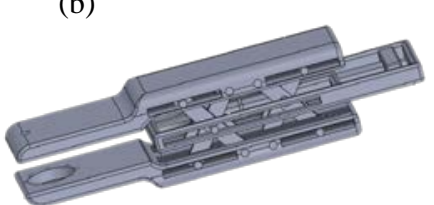

(d)

Fig. 2 Últimos prototipos desarrollados: (a) diagrama del generador, (b) representación en 3D del prototipo, (c) electrodo matricial, y (d) pinza para colocación a una sola mano de electrodos paralelos.

Revista “Jornada de Jóvenes Investigadores del I3A”, vol. 8 (Actas de la IX Jornada de Jóvenes Investigadores del I3A -11 de diciembre de 2020). ISSN 2341-4790. 